\title{
Gene therapy of inherited skin adhesion disorders: a critical overview
}

\author{
M. De Luca, G. Pellegrini and F. Mavilio \\ Centre for Regenerative Medicine Stefano Ferrari, Department of Biomedical Sciences, University of Modena and Reggio Emilia, 41100 Modena, Italy
}

\section{Summary}

Correspondence

Michele De Luca.

E-mail: michele.deluca@unimore.it

Accepted for publication

27 March 2009

Key words

cell therapy, epidermolysis bullosa, gene therapy, skin, stem cells

\section{Conflicts of interest}

None declared.

DOI $10.1111 / j .1365-2133.2009 .09243 . x$
Gene therapy has the potential to treat devastating inherited diseases for which there is little hope of finding a conventional cure. These include lethal diseases, like immunodeficiencies or several metabolic disorders, or conditions associated with a relatively long life expectancy but poor quality of life and expensive and life-long symptomatic treatments, such as muscular dystrophy, cystic fibrosis and thalassaemia. Skin adhesion defects belong to both groups. For the nonlethal forms, gene therapy, or transplantation of cultured skin derived from genetically corrected epidermal stem cells, represents a very attractive therapeutic option, and potentially a definitive treatment. Recent advances in gene transfer and stem cell culture technology are making this option closer than ever. This paper critically reviews the progress and prospects of gene therapy for epidermolysis bullosa, and the technical and nontechnical factors currently limiting its development.
Gene therapy has the potential to treat devastating inherited diseases for which there is little hope of finding a conventional cure. These include lethal diseases, like immunodeficiencies or several metabolic disorders, or conditions associated with a relatively long life expectancy but poor quality of life and expensive and life-long symptomatic treatments, such as muscular dystrophy, cystic fibrosis and thalassaemia. Skin adhesion defects belong to both groups. For the nonlethal forms, gene therapy, or transplantation of cultured skin derived from genetically corrected epidermal stem cells, represents a very attractive therapeutic option, and potentially a definitive treatment. Recent advances in gene transfer and stem cell culture technology are making this option closer than ever. This paper critically reviews the progress and prospects of gene therapy for epidermolysis bullosa (EB), and the technical and nontechnical factors currently limiting its development.

\section{Epidermolysis bullosa}

EB is a family of severe skin adhesion defects due to disruption of the dermal-epidermal junction. EB is classified into simplex, junctional (JEB) and dystrophic (DEB) forms, depending on the level at which the junction is compromised, i.e. above, within or below the basement membrane. ${ }^{1}$ A subclass of JEB is due to autosomal recessive mutations in one of the three chains of laminin 332 (formerly called laminin-5), a key component of the epidermal-dermal junction linking the keratinocyte- specific $\alpha 6 \beta 4$ integrin to the type VII collagen dermal fibrils. Laminin 332 is a heterotrimeric protein made of $\alpha 3, \beta 3$ and $\gamma 2$ chains, encoded by the LAMA3, LAMB3 and LAMC2 genes, respectively. ${ }^{2}$ The severity of laminin 332-deficient JEB can vary from early lethality in the so-called Herlitz variant to much milder conditions in variants characterized by residual gene function. ${ }^{1}$ Most of the JEB mutations occur in 'hot spots' within the LAMB3 gene. In the non-Herlitz forms, the mutations allow the formation of residual, partially functional laminin 332, leading to a phenotype characterized by severe, disfiguring blistering, recurrent infections, visual impairment, and an increased risk of skin cancer. ${ }^{3}$ Collectively, EB affects approximately 20000 individuals in Europe (prevalence: $2 \cdot 83 / 100000$, see http://www.eurordis.org/). There is no cure for EB, and current therapeutic approaches are essentially aimed at controlling infections, protecting the skin from trauma, and maintaining an acceptable quality of life.

\section{The epidermal stem cells}

The continuous renewal of the epidermis is sustained by stem cells contained in the epidermal basal layer and in the bulge of hair follicles. ${ }^{4,5}$ Upon division, epidermal stem cells produce transit amplifying progenitors that generate all the epithelial components of the skin. ${ }^{6-8}$ Epithelial stem cells can be studied at a single-cell level. Clonal analysis of human keratinocytes has identified three types of clonogenic cells, giving rise to 
holoclones, meroclones and paraclones. ${ }^{9}$ The holocloneforming cell is the smallest colony-founding keratinocyte, has the highest proliferative capacity and is the stem cell of the epidermis and the hair follicle. ${ }^{10}$ It soon became clear that the holoclone-forming keratinocyte is the stem cell of virtually all human squamous epithelia. ${ }^{4,5,11,12}$ Holoclones have all the hallmarks of stem cells, including self-renewal capacity, ${ }^{8,10,13}$ telomerase activity and long telomeres, ${ }^{14}$ and an impressive proliferative potential. A single holoclone can indeed generate the entire epidermis or the entire corneal epithelium of a human being. ${ }^{10,12}$ Holoclone-forming cells generate all the epithelial lineages of the tissue of origin, ${ }^{6-8,12}$ permanently restore massive epithelial defects, ${ }^{15-20}$ and can be retrieved from human epidermis regenerated from cultured keratinocytes years after grafting. ${ }^{11}$ During its clonal evolution, the holoclone produces paraclone-forming cells, which have a very limited proliferative capability, generate aborted colonies containing only terminal cells and have the properties expected of transit amplifying cells. ${ }^{9,12}$ Meroclones have an intermediate proliferative and clonogenic potential and are a reservoir of paraclones. ${ }^{9,12}$

Keratinocyte stem cells can be cultivated and expanded in vitro to form transplantable epidermal sheets routinely used for the life-saving treatment of massive full-thickness burns (reviewed in Refs 11, 21). Transplantation of cultured epidermis derived from autologous, genetically corrected epidermal stem cells might therefore be used to replace a substantial fraction of the skin in patients with EB, and possibly provide a definitive cure for this family of diseases.

\section{Gene therapy of epidermolysis bullosa: preclinical investigation}

The first successful attempt to introduce and express foreign genes into transplantable human keratinocytes by retroviral vectors dates back to $1987 .{ }^{22}$ Since then, several groups have shown that genetically modified keratinocytes can express and secrete transgene products, including apolipoprotein-E, clotting factor IX and VIII, and interleukin-6, both in vitro and in vivo in animal models. ${ }^{23-26} \mathrm{~A}$ persistent problem that investigators have faced is the apparent loss of expression of retrovirally encoded transgenes after transplantation in vivo. ${ }^{27,28}$ In most cases, this was ascribed to 'shut-off' of the murine retroviral vector promoter, although failure to transduce stem cells is a just as likely cause of 'unstable' transgene expression, at least in the earliest studies. It soon became clear that the two most important factors to assure persistent expression of retroviral transgenes are the vector design and the use of transduction protocols allowing gene transfer into self-renewing epidermal stem cells. The use of retroviral vectors derived from the MFG prototype, in which the protein of interest is translated from an efficiently spliced genomic RNA under the control of the viral env translation initiation sequences, allowed expression of lacZ or factor IX transgenes for more than 1 year in human epidermal xenografts. ${ }^{26,29}$ Transduction of epidermal stem cells was achieved either by coculture with packaging cell lines $^{25,26,30}$ or by the use of high-titre vector preparations. ${ }^{29}$
Preclinical research carried out in the last decade provided evidence that transduction by retroviral vectors may restore a normal phenotype in keratinocytes obtained from patients affected by JEB or DEB, in culture or upon transplantation on immunodeficient mice. ${ }^{31-33}$ In 1998, Dellambra et al. achieved a high percentage of transduction in epidermal stem cells by coculturing LAMB3-deficient keratinocytes on to a feeder layer composed of lethally irradiated 3T3-J2 cells and packaging cells for a Moloney murine leukaemia virus (MLV)-derived retroviral vector carrying the LAMB3 cDNA under the control of the viral long terminal repeat (LTR) promoter. Gene correction fully restored the keratinocyte adhesion properties and prevented the loss of colony-forming ability, suggesting a direct link between cell adhesion to the basal lamina and keratinocyte proliferative capacity. ${ }^{32}$ Functional proof of correction of the adhesion defect was later provided also in vivo, by transplantation of genetically corrected skin grafts on to immunodeficient mice. ${ }^{33}$

\section{Gene therapy of junctional epidermolysis bullosa: the pilot clinical trial}

Clinical translation of gene therapy of JEB started in Italy in 1999, with the generation of clinical-grade packaging cells and full validation of the gene transfer technology under good manufacturing practice (GMP)/good laboratory practice standards. A phase I clinical trial following the scheme reported in Figure 1 was authorized in June 2002. The trial was aimed at assessing the overall safety of the transduction/transplantation procedure, analysing long-term survival of transduced stem cells and persistence of transgene expression, and monitoring humoral and/or cytotoxic immune responses to the genetically modified cells. The first patient was a 36-year-old man affected by nonlethal JEB caused by double heterozygosity for a LAMB3 null mutation and a single point mutation (E210K), resulting in residual levels of laminin 332 at the level of the basal lamina. To select a donor site suitable for epidermal stem cell correction, skin biopsies were taken from different areas of his body and subjected to keratinocyte clonal analyses. Holoclone-forming cells were undetectable in biopsies from most of the patient's skin, most probably because of the continuous proliferative stimulus associated with the wound healing process. Only palms contained a sufficient number of holoclones.

Keratinocytes obtained from palm skin biopsies were thus transduced at $>95 \%$ efficiency by an MLV-derived retroviral vector carrying the full-length LAMB3 cDNA under the control of the viral LTR, leading to full restoration of laminin 332 synthesis and expression. ${ }^{34}$ Transduced epithelial sheets were grafted on both upper legs in October $2005 .^{34}$ On clinical examination, no blisters were observed in the transplanted area throughout more than 3.5 years of follow up: the regenerated skin is stable, robust and normal looking while the surrounding skin is characterized by the usual chronic blistering lesions. There was no evidence of inflammation, and specific tests carried out 3 and 6 months after transplantation indicated absence of both humoral and $\mathrm{T}$ cell-mediated cytotoxic 
Fig 1. A schematic representation of gene therapy of epidermolysis bullosa by transplantation of genetically corrected skin. Autologous keratinocytes are obtained by a patient's skin biopsy, expanded in culture under conditions that preserve stem cell number and function, transduced by retroviral vectors, grown to produce transplantable epidermal sheets, and transplanted on to the patient dermis after surgical removal of the outer skin layer (Mavilio et al. ${ }^{34}$ ).

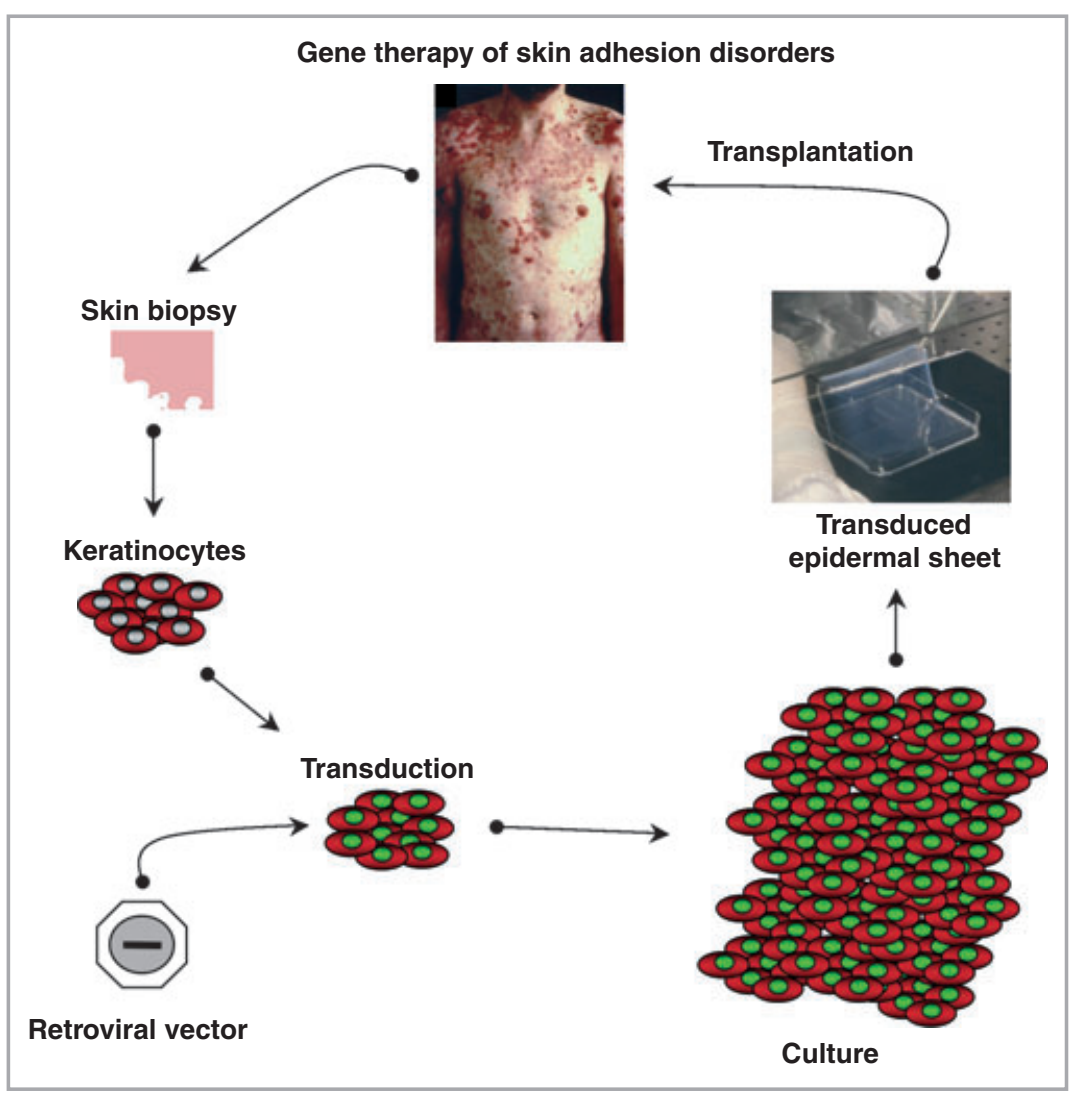

immune response against the transgene product. Skin biopsies taken 1-12 months after grafting showed secretion of laminin 332 heterotrimers, formation of hemidesmosomes and restoration of the epidermal-dermal junction. ${ }^{34}$ A genome-wide analysis of the retroviral integration sites was carried out on DNA extracted from skin biopsies 1 and 4 months after transplantation. Taking the integrated proviruses as a marker of cell clonality, it was estimated that an almost normal repertoire of genetically corrected, long-lasting epidermal stem cells was present in the regenerated epidermis. This study showed that transplantation of autologous cultured epidermis derived from genetically corrected epidermal stem cells is feasible, well tolerated, and leads to long-term functional correction of a skin adhesion defect. ${ }^{34}$

\section{Safety aspects of gene therapy with retroviral vectors}

The pilot clinical trial was based on the use of an MLV-derived retroviral vector. This type of vector has been used in hundreds of clinical trials since 1991. It was considered safe until lymphoproliferative disorders were reported in two patients treated with retrovirally transduced haematopoietic progenitor cells for X-linked severe combined immunodeficiency (X-SCID). ${ }^{35}$ In both cases, the retroviral vector inserted into, and activated, a T-cell proto-oncogene (reviewed in Ref. 36). Since then, three more patients in two different trials of gene therapy of X-SCID developed a similar pathology. ${ }^{37,38}$
Insertional activation of proto-oncogenes has always been considered a possible consequence of random insertion of gene transfer vectors into the genome but, on statistical grounds, the probability of such an event was originally estimated to be less than one in 10 million. ${ }^{39}$ As it turns out, these calculations were based on a wrong assumption: retroviral integration into the human genome is all but random. After the first cases of leukaemia in patients with X-SCID, a number of studies demonstrated that MLV-derived retroviral vectors integrate preferentially into active regions of the genome, and particularly around gene promoters and other regulatory elements. ${ }^{40}$ This 'preference' drastically increases the probability of hitting genes involved in crucial cell functions such as proliferation and differentiation, including proto-oncogenes. ${ }^{41}$ In addition, the viral regulatory elements influence the expression of genes located far away from the insertion points, and at high frequency. ${ }^{42}$ Tumour viruses have most probably evolved these characteristics in order to maximize their ability to transform cells, thereby increasing their chances of being propagated.

This said, the high frequency of leukaemic transformation observed in patients with X-SCID can be only partially explained by the biology of retroviruses, which was unknown when retroviral vectors were developed for medical applications. Indeed, no neoplastic transformation was reported in other clinical trials based on MLV-derived retroviral vectors, such as that for adenosine deaminase-deficient SCID, ${ }^{43}$ suggesting that specific risk factors have contributed to the malignant progression observed in patients with X-SCID. These 
include the nature of the therapeutic gene - a common lymphokine receptor - and accumulation of mutations and chromosome translocations occurring at high frequency in these specific patients.

Despite the lack of evidence that retroviral vector insertion is sufficient per se to promote neoplastic transformation, the results of the X-SCID trials led most regulatory authorities in Europe and the U.S.A. to consider the use of MLV LTR-based vectors as no longer acceptable for genetic modification of stem cells. It can be argued that extrapolating safety concerns from one disease context to another is a rather unusual way of predicting risks, and provides no rationale for putting on hold a promising treatment. This is particularly true for patients with EB, who have very high chances of developing skin cancer anyway. ${ }^{3}$ A promising alternative comes from the development of self-inactivating (SIN), human immunodeficiency virus-derived lentiviral vectors, ${ }^{44}$ which appear to have a potentially more favourable safety profile, based on their integration preferences, ${ }^{40}$ the lack of strong viral enhancers in the LTR, and the lower propensity to generate tumours in preclinical animal models. ${ }^{45}$ Preclinical development of SIN lentiviral vectors specifically targeted to basal layer keratinocytes is in progress, and should provide the next generation of clinical-grade vectors for gene therapy of JEB. ${ }^{46}$ However, developing clinical-grade vectors and assessing their full safety and efficacy profile is going to take several years. In the meantime, patients with no therapeutic alternatives are prevented from accessing a potentially beneficial treatment in the absence of any evidence of potential risks in their specific condition.

\section{Gene transfer vectors: the next generation}

Despite their flexibility and their high efficiency in transducing epidermal stem cells, retroviral vectors, of either gammaretroviral or lentiviral origin, do not yet provide a solution for all EB disorders. In particular, developing a gene transfer strategy for DEB, which is due to the deficiency of the dermal type VII collagen, appears to be a formidable challenge. The COL7A1 cDNA exceeds $9 \mathrm{~kb}$ in length, a size that is hardly accommodated by a retroviral genome. Although there have been successful attempts to produce full-length type VII collagen in human keratinocytes by lentiviral vectors, ${ }^{31}$ titre and genetic stability due to both the size and the highly repeated nature of the cDNA sequence are persistent problems that hamper a real clinical development of this type of vector. To overcome this problem, COL7A1 'minigenes' have been developed which maintain proper biochemical functions in vitro, and could potentially fit into a retrovirus-based gene transfer vector. ${ }^{47}$ However, the efficiency of proteins of reduced size in correcting a collagen deficiency has yet to be demonstrated. Potential alternatives include the use of DNA-based integrating systems, such as those derived by adeno-associated viral integrases, bacteriophage integrases and retrotransposons, or gene correction based on homologous recombination (reviewed in Ref. 48). Although some of these strategies have shown potential in the correction of collagen deficiencies, ${ }^{49}$ none of them allows genetic modification of epidermal stem cells with the efficiency that is necessary for a real clinical translation. Last, but not least, a significant proportion of DEB variants is inherited with a dominant pattern, ${ }^{1}$ a situation in which gene replacement is not going to provide a solution regardless of the efficiency of the gene transfer system. In these cases, gene correction by homologous recombination seems to be the only potential alternative. Homologous recombination has undergone spectacular progress in the recent past, essentially due to the introduction of zinc-finger nuclease technology for targeting specific alleles in the mammalian genome (reviewed in Ref. 50). However, the clinical translation of such technology is still in its infancy, and it will take many years before it will become available to patients.

\section{Cell therapy: a potential alternative for dystrophic epidermolysis bullosa?}

Several studies have recently stressed the possibility of using allogeneic cells as a way of delivering type VII collagen to the dermal-epidermal junction, and therefore as a treatment for DEB. These include local injection of fibroblasts obtained from healthy donors and expanded in culture, ${ }^{51}$ and transplantation of bone marrow-derived haematopoietic stem cells. ${ }^{52}$ However, despite encouraging preclinical or clinical evidence, both strategies have serious biological and practical limitations, and are unlikely to become real therapeutic alternatives. Injected allogeneic fibroblasts synthesize some collagen but are short lived, and most likely cleared by immune rejection. ${ }^{51}$ The situation is reminiscent of the early attempts to treat muscular dystrophy by myoblast injection, which, despite evidence of protein delivery at the right sites, ${ }^{53}$ never progressed as a therapeutic approach due to immune rejection. A possible solution to the problem, i.e. immunosuppression, is unthinkable in patients with severely compromised barrier, such as patients with EB. Preclinical investigation in a mouse model of DEB showed that bone marrow-derived cells can home to damaged skin, produce type VII collagen, ameliorate skin fragility and reduce lethality. Again, this is reminiscent of early attempts to deliver proteins to damaged or dystrophic muscle in mice by bone marrow transplantation, ${ }^{54,55}$ which raised much hope but turned out to be too inefficient to provide real clinical benefit, either in mice ${ }^{56}$ or in humans. ${ }^{57}$ Ongoing clinical trials of bone marrow transplantation in patients with DEB will tell whether conversion of bone marrow cells into skin cells is more efficient than that into muscle cells. Also in this case, however, there are safey issues that should be seriously considered: allogeneic bone marrow transplantation is associated with high morbidity and significant mortality, and is likely to be even more dangerous in patients with high susceptibility to infections and an increased risk of cancer.

\section{Is gene therapy progressing too slowly?}

Despite problems and setbacks, gene therapy has shown a remarkable potential for the therapy of genetic diseases, and 
particularly for skin adhesion defects. Full clinical translation of genetically corrected epidermal stem cells is hampered, however, by a variety of factors, of both economic and regulatory nature. The controversy about the safety of gene therapy technology has elicited enormous attention from scientists, governments and the general public. Reactions from regulatory authorities in the U.S.A. and European countries have ranged from asking for changes in eligibility criteria to provide more safety data to imposing general moratoria on any trial using retroviral vectors. The combination of scientific concern and mixed government reactions has effectively slowed down clinical development of therapies that provided just 'proof-ofprinciple' results. The uncertainty about the medium-term consequences of tighter regulatory frameworks has discouraged scientists from embarking upon new trials, and has scared investors and the biotechnology industry away from the field. This is a particularly serious consequence because in the absence of adequate industrial investment, it is very unlikely that stem cells and gene therapy will deliver generally applicable therapies. The diverse response from regulatory authorities is a crucial uncertainty factor, because it creates a patchwork of different rules in different countries that makes multicentre clinical trials difficult to plan and execute. Harmonization of legislation among European states - and between Europe and the U.S.A. - is urgently needed. In addition, the manufacturing of cell and gene therapeutics must now be carried out under the same rules developed for chemical and biological drugs and only in establishments built and operated within industrial standards and certified by government agencies (GMP). These facilities are too expensive for academic centres to operate or for public grant agencies and charities to fund. This is a serious bottleneck that is turning into a vicious circle: industrial interest will only come when clinical studies demonstrate the efficacy and safety of gene therapy, but in the absence of industrial interest, it is difficult to develop and produce such evidence in the first place. Government agencies, charities, patient organizations and other stakeholders should contribute in developing a new framework to facilitating clinical development of cell and gene therapy technology, while at the same time maintaining the high scientific and ethical standards that are necessary for progress. There are many ways of reaching such goals - from building centralized public facilities to providing public funding to the biotechnology industry. Many scientists argue that cell and gene therapy lie between drug development and organ transplantation, and should be regulated by specific rules, particularly in the early phases of clinical development. Orphan drug and personalized medicine designation should be considered as criteria for developing different standards for manufacturing and carrying out clinical trials. Many of these therapies might never get to the point of seeking formal marketing authorization, and imposing regulation designed for this specific purpose may turn out to be both unnecessary and counterproductive.

The genetic manipulation of stem cells has the vast potential to manage and cure many important diseases. It is in the best interests of our societies to find a way to translate the creativity of so many scientists into a new generation of therapies that could help so many patients live longer and more acceptable lives.

\section{Acknowledgments}

This work was funded by the European Community's 7th Framework Programme, OPTISTEM (grant number 223098), the Regione Emilia Romagna Programma di Ricerca Regione Università 2007/2009 Area 1b 'Medicina Rigenerativa' and the Association Française contre les Myopathies.

\section{References}

1 Epstein EH Jr. The genetics of human skin diseases. Curr Opin Genet Dev 1996; 6:295-300.

2 Aumailley M, Krieg T. Laminins: a family of diverse multifunctional molecules of basement membranes. J Invest Dermatol 1996; 106:209-14.

3 Uitto J, Richard G. Progress in epidermolysis bullosa: from eponyms to molecular genetic classification. Clin Dermatol 2005; 23:33-40.

4 Gambardella L, Barrandon Y. The multifaceted adult epidermal stem cell. Curr Opin Cell Biol 2003; 15:771-7.

5 Blanpain C, Horsley V, Fuchs E. Epithelial stem cells: turning over new leaves. Cell 2007; 128:445-58.

6 Oshima H, Rochat A, Kedzia C et al. Morphogenesis and renewal of hair follicles from adult multipotent stem cells. Cell 2001; 104:233-45.

7 Blanpain C, Lowry WE, Geoghegan A et al. Self-renewal, multipotency, and the existence of two cell populations within an epithelial stem cell niche. Cell 2004; 118:635-48.

8 Claudinot S, Nicolas M, Oshima $\mathrm{H}$ et al. Long-term renewal of hair follicles from clonogenic multipotent stem cells. Proc Natl Acad Sci U S A 2005; 102:14677-82.

9 Barrandon Y, Green H. Three clonal types of keratinocyte with different capacities for multiplication. Proc Natl Acad Sci U S A 1987; 84:2302-6.

10 Rochat A, Kobayashi K, Barrandon Y. Location of stem cells of human hair follicles by clonal analysis. Cell 1994; 76:1063-73.

11 De Luca M, Pellegrini G, Green H. Regeneration of squamous epithelia from stem cells of cultured grafts. Regen Med 2006; 1:45-57.

12 Pellegrini G, Golisano O, Paterna P et al. Location and clonal analysis of stem cells and their differentiated progeny in the human ocular surface. J Cell Biol 1999; 145:769-82.

13 Barbaro V, Testa A, Di Iorio E et al. C/EBPdelta regulates cell cycle and self-renewal of human limbal stem cells. J Cell Biol 2007; 177:1037-49.

14 Dellambra E, Golisano O, Bondanza S et al. Downregulation of 14-3-3sigma prevents clonal evolution and leads to immortalization of primary human keratinocytes. J Cell Biol 2000; 149:111730 .

15 Gallico GG III, O'Connor NE, Compton CC et al. Permanent coverage of large burn wounds with autologous cultured human epithelium. N Engl J Med 1984; 311:448-51.

16 Romagnoli G, De Luca M, Faranda F et al. Treatment of posterior hypospadias by the autologous graft of cultured urethral epithelium. N Engl J Med 1990; 323:527-30.

17 Pellegrini G, Traverso CE, Franzi AT et al. Long-term restoration of damaged corneal surfaces with autologous cultivated corneal epithelium. Lancet 1997; 349:990-3. 
18 Pellegrini G, Ranno R, Stracuzzi G et al. The control of epidermal stem cells (holoclones) in the treatment of massive full-thickness burns with autologous keratinocytes cultured on fibrin. Transplantation 1999; 68:868-79.

19 Ronfard V, Rives JM, Neveux Y et al. Long-term regeneration of human epidermis on third degree burns transplanted with autologous cultured epithelium grown on a fibrin matrix. Transplantation 2000; 70:1588-98.

20 Rama P, Bonini S, Lambiase A et al. Autologous fibrin-cultured limbal stem cells permanently restore the corneal surface of patients with total limbal stem cell deficiency. Transplantation 2001; 72: $1478-85$

21 Pellegrini G, Rama P, Mavilio F, De Luca M. Epithelial stem cells in corneal regeneration and epidermal gene therapy. J Pathol 2009; 217:217-28.

22 Morgan JR, Barrandon Y, Green $\mathrm{H}$ et al. Expression of an exogenous growth hormone gene by transplantable human epidermal cells. Science 1987; 237:1476-9.

23 Fakharzadeh SS, Zhang Y, Sarkar R et al. Correction of the coagulation defect in hemophilia A mice through factor VIII expression in skin. Blood 2000; 95:2799-805.

24 Garlick JA, Katz AB, Fenjves ES et al. Retrovirus-mediated transduction of cultured epidermal keratinocytes. J Invest Dermatol 1991; 97:824-9.

25 Mathor MB, Ferrari G, Dellambra E et al. Clonal analysis of stably transduced human epidermal stem cells in culture. Proc Natl Acad Sci U S A 1996; 93:10371-6.

26 White SJ, Page SM, Margaritis P et al. Long-term expression of human clotting factor IX from retrovirally transduced primary human keratinocytes in vivo. Hum Gene Ther 1998; 9:1187-95.

27 Fenjves ES, Yao SN, Kurachi K et al. Loss of expression of a retrovirus-transduced gene in human keratinocytes. J Invest Dermatol 1996; 106:576-8.

28 Gerrard AJ, Hudson DL, Brownlee GG et al. Towards gene therapy for haemophilia B using primary human keratinocytes. Nat Genet 1993; 3:180-3.

29 Kolodka TM, Garlick JA, Taichman LB. Evidence for keratinocyte stem cells in vitro: long term engraftment and persistence of transgene expression from retrovirus-transduced keratinocytes. Proc Natl Acad Sci U S A 1998; 95:4356-61.

30 Levy L, Broad S, Zhu AJ et al. Optimised retroviral infection of human epidermal keratinocytes: long-term expression of transduced integrin gene following grafting on to SCID mice. Gene Ther 1998; 5:913-22.

31 Chen M, Kasahara N, Keene DR et al. Restoration of type VII collagen expression and function in dystrophic epidermolysis bullosa. Nat Genet 2002; 32:670-5.

32 Dellambra E, Vailly J, Pellegrini G et al. Corrective transduction of human epidermal stem cells in laminin-5-dependent junctional epidermolysis bullosa. Hum Gene Ther 1998; 9:1359-70.

33 Robbins PB, Lin Q, Goodnough JB et al. In vivo restoration of laminin 5 beta 3 expression and function in junctional epidermolysis bullosa. Proc Natl Acad Sci U S A 2001; 98:5193-8.

34 Mavilio F, Pellegrini G, Ferrari S et al. Correction of junctional epidermolysis bullosa by transplantation of genetically modified epidermal stem cells. Nat Med 2006; 12:1397-402.

35 Hacein-Bey-Abina S, von Kalle C, Schmidt M et al. LMO2-associated clonal $\mathrm{T}$ cell proliferation in two patients after gene therapy for SCID-X1. Science 2003; 302:415-19.

36 McCormack MP, Rabbitts TH. Activation of the T-cell oncogene LMO2 after gene therapy for X-linked severe combined immunodeficiency. N Engl J Med 2004; 350:913-22.
37 Hacein-Bey-Abina S, Garrigue A, Wang GP et al. Insertional oncogenesis in 4 patients after retrovirus-mediated gene therapy of SCID-X1. J Clin Invest 2008; 118:3132-42.

38 Howe SJ, Mansour MR, Schwarzwaelder K et al. Insertional mutagenesis combined with acquired somatic mutations causes leukemogenesis following gene therapy of SCID-X1 patients. J Clin Invest 2008; 118:3143-50.

39 Coffin JM, Hughes SH, Varmus HE. Retroviruses. Cold Spring Harbor, NY: Cold Spring Harbor Laboratory Press, 1997.

40 Bushman F, Lewinski M, Ciuffi A et al. Genome-wide analysis of retroviral DNA integration. Nat Rev Microbiol 2005; 3:848-58.

41 Cattoglio C, Facchini G, Sartori D et al. Hot spots of retroviral integration in human CD34+ hematopoietic cells. Blood 2007; 110: $1770-8$.

42 Recchia A, Bonini C, Magnani Z et al. Retroviral vector integration deregulates gene expression but has no consequence on the biology and function of transplanted T cells. Proc Natl Acad Sci U S A 2006; 103:1457-62.

43 Aiuti A, Cassani B, Andolfi G et al. Multilineage hematopoietic reconstitution without clonal selection in ADA-SCID patients treated with stem cell gene therapy. J Clin Invest 2007; 117:2233-40.

44 Zufferey R, Dull T, Mandel RJ et al. Self-inactivating lentivirus vector for safe and efficient in vivo gene delivery. J Virol 1998; 72:9873-80.

45 Montini E, Cesana D, Schmidt M et al. Hematopoietic stem cell gene transfer in a tumor-prone mouse model uncovers low genotoxicity of lentiviral vector integration. Nat Biotechnol 2006; 24:687-96.

46 Di Nunzio F, Maruggi G, Ferrari S et al. Correction of laminin-5 deficiency in human epidermal stem cells by transcriptionally targeted lentiviral vectors. Mol Ther 2008; 16:1977-85.

47 Chen M, O’Toole EA, Muellenhoff $\mathrm{M}$ et al. Development and characterization of a recombinant truncated type VII collagen 'minigene'. Implication for gene therapy of dystrophic epidermolysis bullosa. J Biol Chem 2000; 275:24429-35.

48 Porteus MH, Connelly JP, Pruett SM. A look to future directions in gene therapy research for monogenic diseases. PLoS Genet 2006; 2:e133.

49 Ortiz-Urda S, Thyagarajan B, Keene DR et al. Stable nonviral genetic correction of inherited human skin disease. Nat Med 2002; 8:116670.

50 Porteus $\mathrm{MH}$. Mammalian gene targeting with designed zinc finger nucleases. Mol Ther 2006; 13:438-46.

51 Wong T, Gammon L, Liu L et al. Potential of fibroblast cell therapy for recessive dystrophic epidermolysis bullosa. J Invest Dermatol 2008; 128:2179-89.

52 Tolar J, Ishida-Yamamoto A, Riddle $\mathrm{M}$ et al. Amelioration of epidermolysis bullosa by transfer of wild-type bone marrow cells. Blood 2009; 113:1167-74.

53 Gussoni E, Blau HM, Kunkel LM. The fate of individual myoblasts after transplantation into muscles of DMD patients. Nat Med 1997; 3:970-7.

54 Ferrari G, Cusella-De Angelis G, Coletta M et al. Muscle regeneration by bone marrow-derived myogenic progenitors. Science 1998; 279: 1528-30.

55 Gussoni E, Soneoka Y, Strickland CD et al. Dystrophin expression in the $\mathrm{mdx}$ mouse restored by stem cell transplantation. Nature 1999; 401:390-4.

56 Ferrari G, Stornaiuolo A, Mavilio F. Failure to correct murine muscular dystrophy. Nature 2001; 411:1014-15.

57 Gussoni E, Bennett RR, Muskiewicz KR et al. Long-term persistence of donor nuclei in a Duchenne muscular dystrophy patient receiving bone marrow transplantation. J Clin Invest 2002; 110:807-14. 